\title{
Investigating the use of a lecture capture system within pharmacy education: Lessons from an internationally accredited undergraduate pharmacy program
}

\author{
- Farhat Naz HUSSAIN ${ }^{1}$, Reem AL-MANNAI ${ }^{1}$, Mohammad Issam DIAB ${ }^{1}$, Abdelali AGOUNI ${ }^{1}$ \\ ${ }^{1}$ College of Pharmacy, QU Health, Qatar University
}

\section{INTRODUCTION}

- Multiple technologies are currently used to assist teaching and introduce active learning in the classroom.

- Lecture capture has a variety of benefits (e.g. students choose the location, pace and frequency of studying).

- Video lecture capture was introduced at our institution nearly a decade ago.

\section{AIM OF THE STUDY}

- Evaluate lecture capture viewings for professional undergraduate pharmacy courses in the Fall and Spring semesters over three consecutive academic years.

- Analyze longitudinally the data for one cohort (class of 2020) for six consecutive academic semesters.

\section{METHODS}

1. Viewing figures downloaded and analyzed for courses in three consecutive academic years (16AY, 17AY, 18AY).

2. Inclusion criteria: Courses delivered in series between the Fall and Spring semesters and had at least two years of data available.

3. Exclusion criteria: Standalone courses that did not occur as a series, those that did not have enough data, or those in which the nature of the course rendered it unsuitable for recording (e.g. lab-based courses).

\section{RESULTS and DISCUSSION}

Figure 1. Viewing pattern for professional year 1 courses

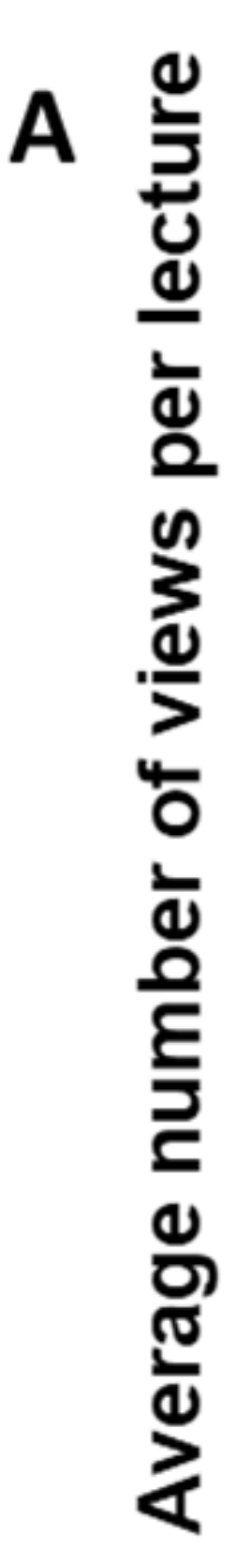

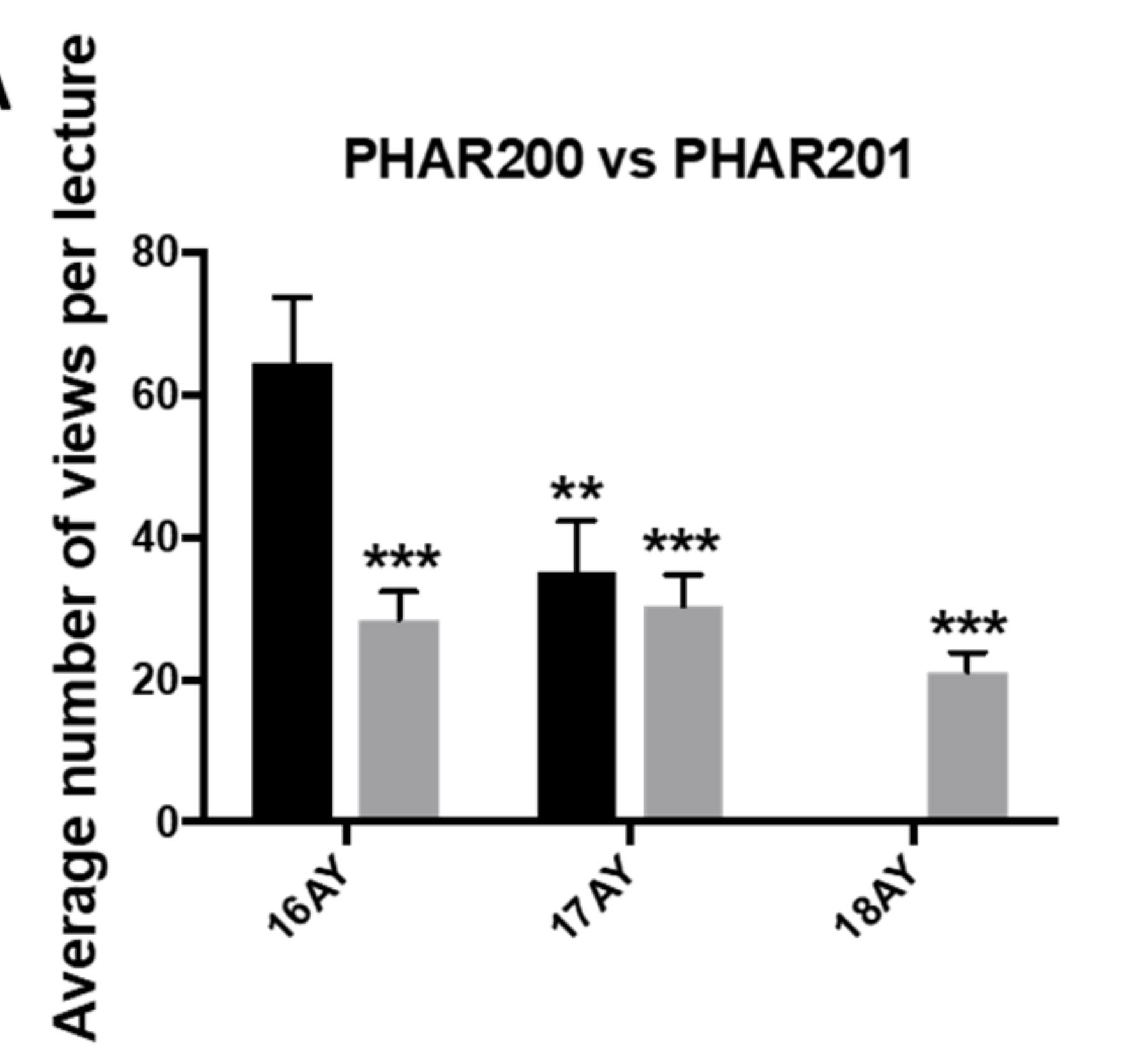

C

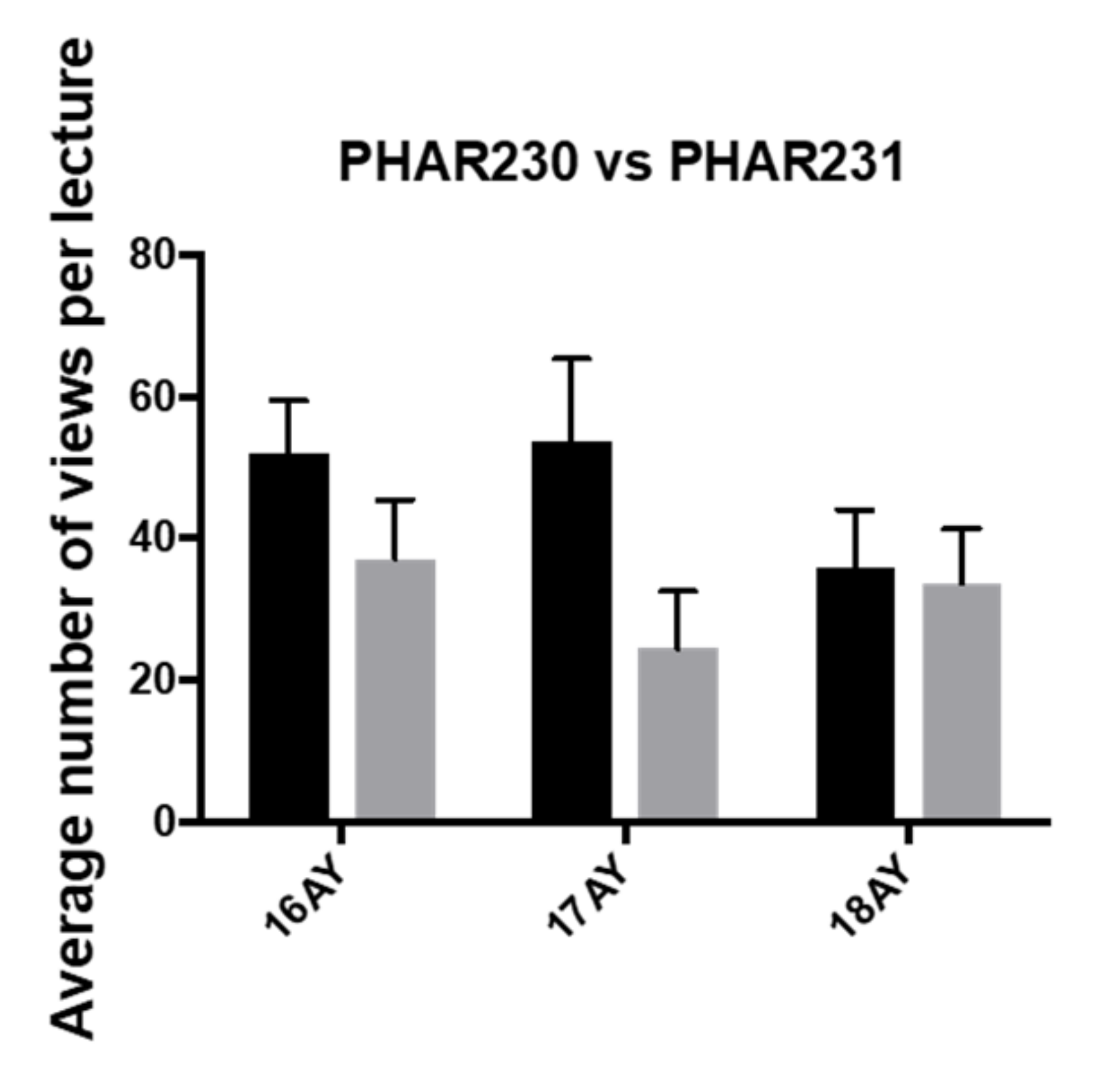

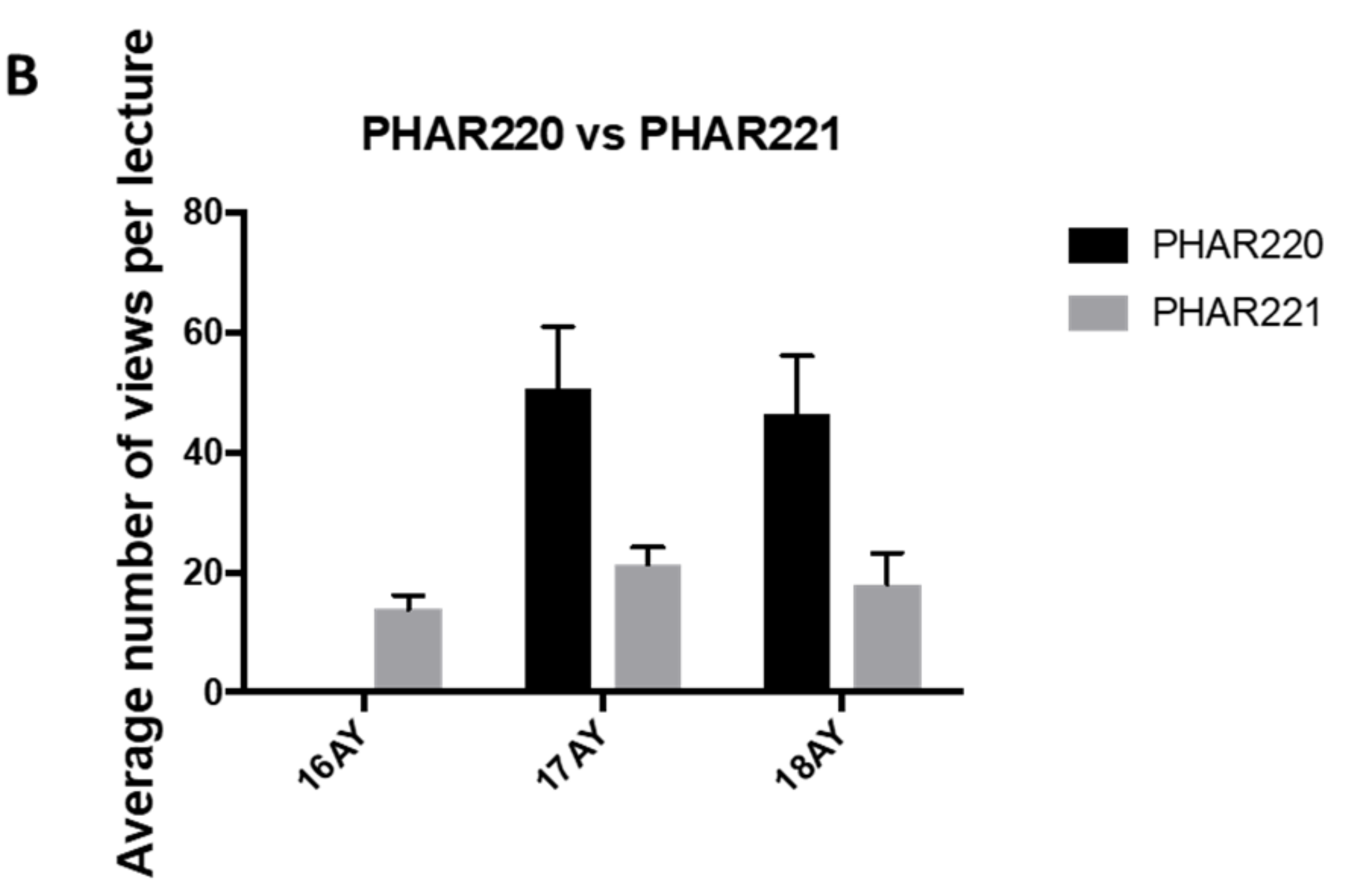

- A higher number of views was observed throughout the Fall semester in professional year 1 (black bars) and across different professional years; however, a decrease in number of views was evident for the consecutive courses during the Spring semester (grey bars).
Figure 2. Viewing pattern for Class of 2020 throughout three academic years

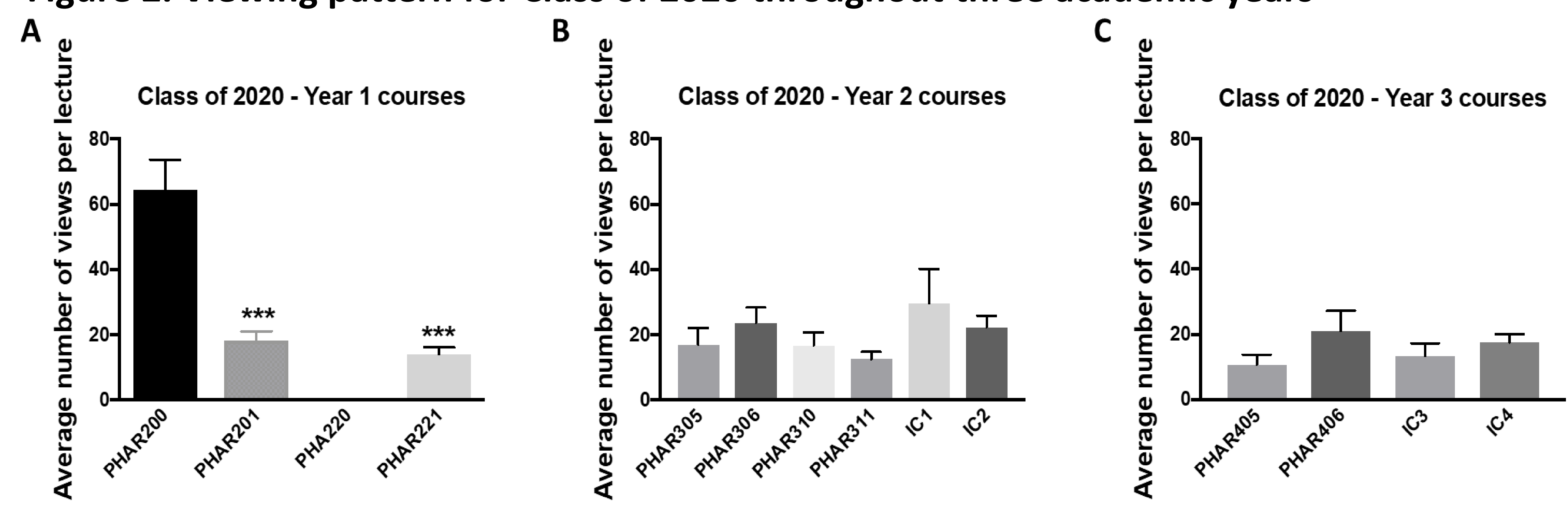

- The P1-PHAR 200 course showed the highest viewing figures (average of 65) compared to all other courses, whereas P2 and P3 viewing figures remained consistant (less than 30).

- Within P1 and P2, viewing figures declined from the Fall to Spring semester; however, for this cohort, P3 course viewing figures showed a trend for increase from the Fall to Spring semester.

\section{CONCLUSION AND FUTURE DIRECTIONS}

- Our findings suggest that the lecture capture system within our College is primarily utilized by junior pharmacy students. Further studies must be conducted to fully understand why.

- Additional quantitative and qualitative studies must be conducted to fully grasp the motivations for use, attitudes and perceptions towards the system.

- A mixed methods study (questionnaire and focus group) with both students and faculty may help us in devising a bespoke action plan to tackle the identified barriers for a wider use of this technology. 